\title{
DIFFERENTIABILITY OF HÖLDER-CONTINUOUS SEMIGROUPS
}

\section{WAELBROECK ${ }^{1}$}

1. A semigroup is a mapping $u: R_{+} \rightarrow A$ of the positive half line into an algebra with unit such that $u(0)=1, u(s+t)=u(s) u(t)$. We assume that $A$ is a $b$-algebra. The semigroup is differentiable if $u$ is a differentiable mapping of $\boldsymbol{R}_{+}$into $A$. It is Hölder-continuous if some $p>0$ exists such that $(u(t)-1) / t^{p}$ is bounded on some neighborhood of zero. We shall show that a Hölder-continuous semigroup is differentiable.

For the definition of $b$-spaces and $b$-algebras, and of differentiable mappings into $b$-spaces and $b$-algebras, the reader is referred to [2] and [3]. We shall use the standard convergence structure of a $b$-space. If $E$ is a $b$-space, and $e_{k}$ are elements of $E$, where $k$ ranges over a directed system, $e_{k} \rightarrow e$ if $k_{0}$ and a completant bounded $B$ can be found such that $e_{k} \in E_{B}$ for $k \geqq k_{0}, e_{k} \rightarrow e$ in the norm topology of $E_{B}$. The $O$-notation is also standard; if $e_{k}$ are elements of $E$ and $c_{k}$ are scalar, $e_{k}=O\left(c_{k}\right)$ if a bounded system of $e_{k}^{\prime}$ can be found such that $e_{k}=c_{k} e_{k}^{\prime}$.

2. The infinitesimal generator of the semigroup $u$ is the limit (if it exists)

$$
a=\lim _{t \rightarrow 0} \frac{u(t)-1}{t} .
$$

The proof that a semigroup is differentiable if and only if it has an infinitesimal generator will be left to the reader.

The infinitesimal generator determines the semigroup, has the same commutant as the semigroup, the equation $f^{\prime}=$ af has a unique solution $\boldsymbol{R}_{+} \rightarrow A$ which takes a given initial value $f(0)$ if a generates a semigroup, this solution is given by $f(t)=u(t) f(0)$.

These results are easy to prove. We consider first a solution of the equation $f^{\prime}=a f$ and differentiate $u(s) f(t-s)$ with respect to $s$. The derivative vanishes, hence $u(t) f(0)=u(0) f(t)=f(t)$, as required. A differentiable semigroup having $a$ as infinitesimal generator is a solution of the equation $u_{1}^{\prime}=a u_{1}$ with initial value $u_{1}(0)=1$ but the solution is unique and $u$ is a solution. An element $b$ clearly commutes with $a$ if it commutes with all values of $u(s)$. If conversely $a b=b a$,

\footnotetext{
Received by the editors November 7, 1967 and, in revised form, April 19, 1968.

1 Supported in part by the National Science Foundation under grant number
} GP-5683. 
put $f(t)=b u(t)$, then $f^{\prime}=b u^{\prime}=b a u=a b u=a f$, and $f(t)=u(t) f(0)$, i.e. $b u(t)=u(t) b$.

The reader is referred to [1], where an apparently useless necessary and sufficient condition is given for an element $a$ to generate a differentiable semigroup.

3. The proof of the differentiability of the semigroup considered uses identities involving the function $v$ defined for $t>0$ by

$$
v(t)=(u(t)-1) / t, \quad u(t)=1+t v(t) .
$$

From $u(t)^{2}=u(2 t)$, substituting for $u$ its expression in function of $v$, simplifying, we obtain

$$
v(2 t)-v(t)=(t / 2) v(t)^{2} .
$$

From $u(t)^{3}=u(3 t)$ we obtain

$$
v(3 t)-v(t)=t v(t)^{2}+\left(t^{2} / 3\right) v(t)^{3} .
$$

4. The boundedness of $w(t)=[u(t)-1] / t^{p}$ on some interval $(0, \epsilon)$ implies that of $w$ on all finite intervals $(0, M)$. As a matter of fact $u(t)=1+t^{p} w(t)$ is then bounded on $(0, \epsilon)$. Because $u(t)=u(t / 2)^{2}$ we see that $u$ is also bounded on $(0,2 \epsilon)$, and by induction on all finite intervals $(0, M)$. Furthermore, $t^{-p}$ is bounded on $[\epsilon, M)$; therefore $w(t)=t^{-p}[u(t)-1]$ also, and hence on $(0, \epsilon) \cup[\epsilon, M)$. Similarly (letting $p=1)$, the boundedness of $v(t)=[u(t)-1] / t$ on some interval $(0, \epsilon)$ implies that $v$ is bounded on $(0, M)$.

5. The first step will be the proof that a Hölder-continuous semigroup is Lipschitz-continuous. We shall assume that $[u(t)-1] / t^{p}$ is bounded near the origin and prove that $(u(t)-1) / t$ is bounded there.

Choose $k$ in such a way that $1 \leqq 2^{k} t<2, k$ is a function of $t, k$ $=O(-\log t), t=O\left(2^{-k}\right)$. Applying (2) inductively we obtain

$$
v\left(2^{k} t\right)-v(t)=\sum_{0}^{k-1} 2^{n-1} t v\left(2^{n} t\right)^{2}
$$

We want to evaluate $v(t)$, assuming that $v(t)=O\left(t^{p-1}\right)$. In the relation (4), $v\left(2^{k} t\right)$ is bounded because $v$ is bounded on the interval $(1,2)$. In the expression on the right-hand side $2^{n-1} t=O\left(2^{n-k}\right), v\left(2^{n} t\right)=$ $O\left(2^{(n-k)(p-1)}\right)$ and

$$
2^{n-1} t v\left(2^{n} t\right)^{2}=O\left(2^{(k-n)(1-2 p)}\right) .
$$

This gives $v\left(2^{k} t\right)-v(t)=O\left(\sum_{0}^{k-1} 2^{(k-n)(1-2 p)}\right)$.

We remember that $v\left(2^{k} t\right)$ is bounded for all choices of $t<1$. 
If $p<1 / 2, v(t)=O\left(2^{k(1-2 p)}\right)=O\left(t^{2 p-1}\right)$.

If $p=1 / 2, v(t)=O(k)=O(-\log t)$.

If $p>1 / 2, v(t)=O(1)$.

In other words, we have shown that $u(t)-1=O\left(t^{2 p}\right)$ if $u(t)-1$ $=O\left(t^{p}\right)$ and $p<1 / 2$, we have shown that $u(t)-1=O(t)$ if $u(t)-1$ $=O\left(t^{p}\right)$ and $p>1 / 2$. If $p=1 / 2$, we have shown that $u(t)-1=O(t \log t)$ and $t \log t=O\left(t^{1-\epsilon}\right)$ for all $\epsilon>0$. This is sufficient to prove the statement.

6. Let $u$ be a Lipschitz semigroup; a bounded completant $B$ can be found such that $v\left(2^{-k} t\right)$ and $v\left(3^{-k} t\right)$ both converge as $k \rightarrow \infty$ in the normed space $A_{B}$, the convergence being uniform for $t<1$.

This will follow from relations (2), (3). The set $B$ will be chosen bounded completant and such that $u(t), v(t), v(t)^{2}, v(t)^{3}$ all belong to $B$ for $t<1$.

$$
\begin{aligned}
& v\left(2^{-k} t\right)-v\left(2^{-k-1} t\right)=2^{-k-2} t v\left(2^{-k-1} t\right)^{2} \in 2^{-k} B \\
& v\left(3^{-k} t\right)-v\left(3^{-k-1} t\right)=3^{-k-1} t v\left(3^{-k-1} t\right)^{2}+3^{-k-3} t^{2} v\left(3^{-k-1} t\right)^{3} \in 3^{-k} B .
\end{aligned}
$$

This proves the uniform convergence of $v\left(2^{-k} t\right)$ and $v\left(3^{-k} t\right)$ in the Banach space $A_{B}$.

7. We finally observe that $f$ tends to a limit as $x$ tends to infinity if $E$ is a Banach space, $f$ a continuous E-valued function on $\boldsymbol{R}_{+}$and if $f(x+k), f(x+k h)$ both converge uniformly as $k$ tends to infinity by integral values, where $h$ is irrational.

This is sufficient to prove that $v(x)$ tends to a limit as $x$ tends to zero. The proof is simple. We include it for completeness sake.

Let $g_{1}(x)=\lim f(x+k), g_{1}$ is continuous and has the period 1. The difference $f-g_{1}$ tends to zero as $x$ tends to infinity. As a matter of fact if $x>M, x^{\prime}=x-k_{0}>0$ for some large $k_{0}$. Because of uniform convergence $f\left(x^{\prime}+k_{0}\right)-g_{1}\left(x^{\prime}\right)=f(x)-g_{1}(x)$ is small.

Similary $g_{h}(x)=\lim f(x+k h)$ is continuous, has the period $h$, and $f-g_{h}$ tends to zero as $x$ tends to infinity. And $g_{1}-g_{h}$ is a continuous almost periodic function which converges to zero at infinity, $g_{1}=g_{h}$ $=$ constant.

$f$ tends to that constant since $f-g_{1}$ tends to zero.

8. By way of application we can prove the following result: If $f$ is a function on $R_{+}$, we put $\Delta_{h} f(x)=f(x+h)-f(x)$. Let $E$ be the space of functions on $\boldsymbol{R}_{+}$such that for all values of $k$ and some positive $p$

$$
\left(\Delta_{h_{1}} \cdots \Delta_{h_{k}} f(x)\right) /\left(h_{1}^{p} \cdots h_{k}^{p}\right)
$$


is a locally bounded function on $R_{+}^{k+1}$. Topologise $E$ by uniform convergence of all expressions (5) on compact subsets of $R_{+}^{k+1}$. Then $E$ is the space $E\left(R_{+}\right)$of all $C_{\infty}$ functions on $R_{+}$, topologised by uniform convergence of the function and all its derivatives.

It is clear that $E\left(R_{+}\right) \subseteq E$ and that the identity map is continuous. We must prove the converse of that statement. We define a semigroup in the ring of continuous linear transformations by $E$ by putting $u_{h} f(x)=f(x+h)$. This is clearly a Hölder semigroup when we put on the ring considered the equicontinuous boundedness. This is therefore a differentiable semigroup.

Let $p \in E^{*}$ be evaluation at the origin, $\langle f, p\rangle=f(0)$. Then $\left\langle u_{h} f, p\right\rangle$ $=f(h)$ is a differentiable function of $h$. This shows $E \subseteq E\left(R_{+}\right)$.

The infinitesimal generator $a$ of the semigroup $u$ is clearly the derivation operator. The set of operators $a^{r} u_{h}(0 \leqq h \leqq M)$ is an equicontinuous set of transformations of $E$, hence $\left\langle a^{r} u_{h} f_{n}, p\right\rangle=f_{n}{ }^{(r)}(h) \rightarrow 0$ uniformly on $(0, M)$ if $f_{n} \rightarrow 0$. This ends the proof.

\section{BIBLIOGRAPHY}

1. L. Waelbroeck, Les semi-groupes differentiables, Deuxième Colloq. Analyse Fonctionelle, Centre Belge Recherches Math., Librairie Universitaire, Louvain, 1964, pp. 97-103.

2. - Some theorems on bounded structures, J. Functional Analysis 1 (1967), 392-408.

3. , Differentiable mappings into b-spaces, J. Functional Analysis 1 (1967), 409-418.

MASSAChUSETtS INSTITUTE OF TECHNOLOGY 